\title{
Estimations of uncertainties of frequencies
}

\author{
Laurent Eyer ${ }^{1}$, Jean-Marc Nicoletti ${ }^{2}$ and Stephan Morgenthaler ${ }^{3}$ \\ ${ }^{1}$ Department of Astronomy, University of Geneva, CH-1290, Sauverny, Switzerland \\ email: Laurent.Eyer@unige.ch \\ ${ }^{2}$ Swiss Statistics Federal Office, CH-2010 Neuchâtel, Switzerland \\ ${ }^{3}$ Swiss Federal Institute of Technology, EPFL, CH-1015 Lausanne, Switzerland
}

\begin{abstract}
Diverse variable phenomena in the Universe are periodic. Astonishingly many of the periodic signals present in stars have timescales coinciding with human ones (from minutes to years). The periods of signals often have to be deduced from time series which are irregularly sampled and sparse, furthermore correlations between the brightness measurements and their estimated uncertainties are common. The uncertainty on the frequency estimation is reviewed. We explore the astronomical and statistical literature, in both cases of regular and irregular samplings. The frequency uncertainty is depending on signal to noise ratio, the frequency, the observational timespan. The shape of the light curve should also intervene, since sharp features such as exoplanet transits, stellar eclipses, raising branches of pulsation stars give stringent constraints. We propose several procedures (parametric and nonparametric) to estimate the uncertainty on the frequency which are subsequently tested against simulated data to assess their performances.
\end{abstract}

Keywords. methods: statistical, data analysis, stars: variables, planetary systems

\section{Summary}

The estimation of uncertainties of frequency has been studied from the statistics side (e.g. Whittle 1952, Walker 1971, Walker 1973) and astronomy side (e.g. Kovacs 1981, Cuypers 1987). For a sinusoidal curve, the uncertainty on the frequency is inversely proportional to the signal to noise ratio, the time span of the observations, and the square root of the number of observations. Hall et al. (2000) took into account the light curve shape for this estimation, it is with IID (Independent and Identically Distributed) errors and an irregular sampling. A generalisation was made by Nicoletti (2012), where the hypothesis of IID is relaxed. An application to a box signal (for exoplanet transits) has been studied and asymptotic behaviour are derived. As for the signal sinusoidal the frequency uncertainty is inversely proportional to the signal to noise ratio (depth of transit/measurement error), the time span and differently from the sine-shape the number of measurements. The next steps of our studies are: (1) From simulations, to determine the boundaries of the validity of the asymptotic behaviour, (2) We will derive, if possible, ad-hoc corrections when the signal has a small number of measurements.

\section{References}

Cuypers, J. 1987, Acad. Analecta, 49, 3

Nicoletti, J.-M. 2012, PhD Thesis 5296 of EPFL

Kovacs, G. 1981, ApछSSS, 78, 175

Hall, P., Reimann, J., \& Rice, J. 2000, Biometrika, 87, 545

Walker, A. M. 1971, Biometrika, 58, 21

Walker, A. M. 1973, Advances in Applied Probability, 5, 217

Whittle, P. 1952, Biometrika, 39, 309 